\title{
Hyperboloidal data and evolution
}

\author{
S. Husa ${ }^{*}$, C. Schneemann ${ }^{\dagger}$, T. Vogel ${ }^{\dagger}$ and A. Zenginoğlu ${ }^{\dagger}$ \\ ${ }^{*}$ Friedrich Schiller University Jena \\ ${ }^{\dagger}$ MPI for Gravitational Physics Potsdam
}

\begin{abstract}
We discuss the hyperboloidal evolution problem in general relativity from a numerical perspective, and present some new results. Families of initial data which are the hyperboloidal analogue of Brill waves are constructed numerically, and a systematic search for apparent horizons is performed. Schwarzschild-Kruskal spacetime is discussed as a first application of Friedrich's general conformal field equations in spherical symmetry, and the Maxwell equations are discussed on a nontrivial background as a toy model for continuum instabilities.
\end{abstract}

Keywords: Numerical relativity, continuum instabilities, initial data, conformal compactification PACS: 04.25.Dm, 04.20.Ha, 04.30.-w

\section{INTRODUCTION}

In this paper we consider algorithms for numerical relativity (NR) based on hyperboloidal slices - spacelike hypersurfaces characterized by a mean extrinsic curvature $\chi$ that approaches a finite value in the limit $r \rightarrow \infty$. Correspondingly such slices are not asymptotically euclidean, but rather reach out to null infinity, and thus provide an alternative to null surfaces for tracking radiation signals to large distances from their source, e.g. in order to predict signals in a gravitational wave detector (see e.g. [1, 2]). Being spacelike, hyperboloidal slices are in some sense more flexible than null surfaces, and thus interesting for constructing numerical relativity codes aimed at gravitational wave physics. In this paper we briefly discuss our general ideas about the design of numerical codes for hyperboloidal evolution and some preliminary results from two perspectives we believe to be of key importance: the need to control continuum instabilities and fitness to accurately resolve gravitational wave signals. We also list a few new results.

As is the case with other disciplines of computational physics, an essential part of the art of NR is to make the physical continuum features manifest in the discrete system that is then solved by a computer. In general relativity (GR) diffeomorphism invariance gives rise to a variety of problems not familiar from other theories and which are not yet understood in sufficient depth to provide a fully satisfactory basis for numerical simulations. A typical evolution scheme has many more computational than physical degrees of freedom, the extra degrees of freedom correspond to gauge choice and the presence of constraints - one should therefore not be surprised to find a generic tendency for instabilities in the excess degrees of freedom, and indeed the multitude of formulations of the Einstein equations are typically plagued by instabilities whose precise causes have often remained elusive, and we expect much further work to be necessary in order to understand the dos and don'ts of NR. As a simple (linear) example for the type of problems that have to be expected, we will consider hyperboloidal

\footnotetext{
CP841, A Century of Relativity Physics: XXVIII ${ }^{\text {h }}$ Spanish Relativity Meeting,

edited by L. Mornas and J. Diaz Alonso

(C) 2006 American Institute of Physics 0-7354-0333-3/06/\$23.00
} 
evolution of an electromagnetic field on Minkowski background.

At least from an observational point of view it is clearly desirable to design numerical codes with accurate GW signal prediction in mind. This is difficult for various reasons. First, in all physically relevant scenarios gravitational radiation is only a relatively small effect in the energy balance of the system. Second, in GR such fundamental quantities as energy, momentum, or emitted gravitational radiation energy can only be defined unambiguously in terms of asymptotic limits. Consequently, it also becomes particularly difficult to formulate physically motivated boundary conditions along the lines of "outgoing radiation boundary conditions" at finite distance from the sources.

Conformal compactification, originally suggested by Penrose [3] allows to discuss asymptotics in terms of local differential geometry and has provided a very fruitful framework to approach many problems in mathematical relativity. Naturally, it also raises hopes for a consistent notion and quantitative treatment of GW signals. However, since the conformal framework is extremely flexible, it does not by itself determine a strategy for NR, and additional physical intuition and practical insights are necessary to bring this technique to fruition in numerical simulations. In the following, we will briefly review the connection between asymptotics, conformal compactification and gravitational wave $(\mathrm{GW})$ signals before sketching our strategy to develop codes for the hyperboloidal evolution problem. We then present some new numerical results concerning Friedrich's general conformal field equations in spherical symmetry as a simple window into the interplay of spatial and null infinity, the Maxwell equations on a nontrivial background as a toy model for continuum instabilities, and initial data that generalize Brill waves to the hyperboloidal context.

\section{CONFORMAL COMPACTIFICATION AND RESCALING}

A key idea behind conformal rescaling is to compute "order unity" quantities, e.g. for a massless scalar field $\Phi$ a rescaling of the type $\Psi:=r \Phi$, which asymptotically just cancels the known fall-off of the radiation from an isolated source. This allows one to work with quantities that are finite even asymptotically. Such a procedure can furthermore improve the numerical conditioning of radiation problems. Generally, it is useful in computational work to factor out what is already known. The idea of conformal compactification is to perform a conformal transformation on the metric $g_{a b}=\Omega^{2} \tilde{g}_{a b}$ and view the physical space-time $\tilde{\mathcal{M}}$ as a submanifold $\tilde{\mathcal{M}}=\{p \in \mathcal{M} \mid \Omega(p)>0\}$ of some manifold $\mathcal{M}$ completed by boundary points $\partial \tilde{\mathcal{M}}=\{p \in \mathcal{M} \mid \Omega(p)=0\}$ lying "at infinity" with respect to $\tilde{g}_{a b}$. The definition of a certain type of asymptotics, like asymptotic flatness, then proceeds in terms of asymptotic properties of the conformal factor, which define a desired physical fall-off behavior (see e.g. [1]). Note that in a relativistic theory, we need to deal with three types of directions toward infinity: timelike $\left(l^{ \pm}\right)$, spacelike $\left(l^{0}\right)$ and null $\left(\mathscr{I}^{ \pm}\right)$, and these limits have very different physical significance. In particular, observers situated at "astronomical" distances (e.g. GW detectors) can be modeled through geometric objects at future null infinity [4]. Clearly, a thorough physical understanding of the problem of consistently modeling GW sources and detectors in a single picture is very desirable. 
However, writing the Einstein tensor in terms of the rescaled metric makes it immediately clear that taking this concept to the level of the field equations can not be straightforward:

$$
\tilde{G}_{a b}\left[\Omega^{-2} g\right]=G_{a b}[g]-\frac{2}{\Omega}\left(\nabla_{a} \nabla_{b} \Omega-g_{a b} \nabla_{c} \nabla^{c} \Omega\right)-\frac{3}{\Omega^{2}} g_{a b}\left(\nabla_{c} \Omega\right) \nabla^{c} \Omega .
$$

In the new variables the equations are formally singular at $\partial \tilde{\mathcal{M}}$ whereas multiplication by $\Omega^{2}$ leads to a degenerate principal part for $\Omega=0$. A very general prescription for regularizing the rescaled Einstein equations has been obtained by Friedrich through the formulation of the regular conformal field equations [5]. The fact that this is actually possible for the Einstein equations, is a nontrivial result and may certainly seem surprising. Unfortunately, it is achieved at a high price of introducing a large number of new evolution variables, which complicates the numerical implementation and increases the risk of triggering continuum instabilities (for numerical results see [2]).

Compactification techniques have been used in NR for quite some time, but have often been based on less general regularization techniques, e.g. through restriction to a special class of gauges. Compactification in null directions has been very successful in the characteristic approach (see e.g. [6] and [7] for recent results) and is well understood. Compactification of spacelike infinity has not only been used to construct initial data (see e.g. $[8,9,10])$, but encouraging results have also been obtained in the time evolution problem [11], where black holes are modeled as "internal asymptotic ends", often referred to as punctures, and recently also to get rid of the boundary problem in NR [12]. In the evolution context, however, some open questions remain, e.g. because compactification at $\iota^{0}$ leads to a "piling up" of waves. At $\mathscr{I}^{+}$this effect does not appear - waves leave the physical spacetime through the boundary $\mathscr{I}^{+}$. Also, regularity issues of the equations at spatial infinity are not yet fully understood, although much progress has been made with Friedrich's general conformal field equations [13], for which we discuss a simple application below.

A code that utilizes hyperboloidal slices to compactify null infinity can profit from all the flexibility in gauge that a Cauchy approach offers. However, following the idea to factor out what is already known and making the physical continuum features also manifest in the numerical code leads to the problem of making manifest the rigid structure of null infinity in addition to the fall-off of the "gravitational field". Particularly important seem the shear-free property of null infinity and the existence of a natural class of time coordinates associated with affine parameters of the null geodesic generators of $\mathscr{I}$, known as Bondi time. It is this time coordinate which corresponds to the proper time of distant observers [1], and which thus corresponds to an "undistorted signal", as in Fig. 1. We suggest to use the gauge freedom to make the rigid structure of $\mathscr{I}$ manifest and freeze it to a fixed coordinate sphere as discussed in detail by Andersson [14] (in particular here the connection between the $3+1$ split and the Bondi gauge is discussed, and essentially the same recommendation to use such a gauge as starting point for regularization is given). Fixing $\mathscr{I}^{+}$to a coordinate sphere, it is natural to identify it also with the boundary of the computational domain, and thus to restrict oneself to the physical part of the spacetime. First experiments along these lines with scalar fields on a Schwarzschild background have yielded the ringdown results in Fig. 1. 
As an example for the type of coordinates we have in mind, consider computing just the domain of dependence of a piece of Minkowski space with initial data given on a ball. Appropriate coordinates are those which are also adapted to self-similarity:

$$
d s^{2}=\frac{e^{-2 \tau}}{R^{2}}\left[-\left(R^{2}-r^{2}\right) d \tau^{2}-2 r d r d \tau+d r^{2}+r^{2}\left(d \theta^{2}+\sin \theta^{2} d \varphi^{2}\right)\right] .
$$

Using the same type of coordinates in the compactified spacetime with $R$ identified with the initial location of $\mathscr{I}$ yields the picture in Fig. 1 . Freezing $\mathscr{I}$ to a coordinate sphere essentially corresponds to a choice of the shift vector on $\mathscr{I}$, which leads to two problems: First, the prescription of shift needs to be compatible with a well-posed evolution system, and second, one also needs to choose well for the shift vector away from $\mathscr{I}$, in order not to distort the geometry in the interior of the spacetime.
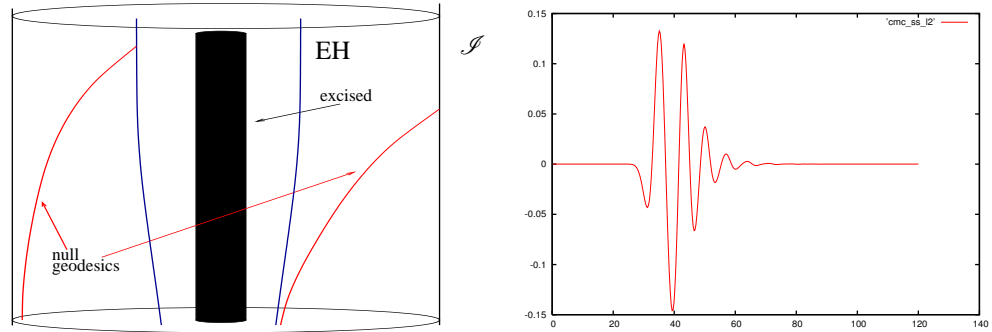

FIGURE 1. Left: A sketch depicting a situation with $\mathscr{I}^{+}$frozen to a coordinate sphere. Right: The ringdown of a scalar field with angular momentum number $l=2$ on a compactified Schwarzschild background with CMC-slices and $\mathscr{I}^{+}$frozen to a coordinate sphere.

Our strategy to develop codes for the hyperboloidal initial value problem has thus been threefold: First, we have developed a computational infrastructure that allows us to confront equations as complex as the conformal field equations without tying us down to a particular form of the equations. To this end, the Kranc code generation and tensor manipulation package [15] has been developed. Second, it has proven very fruitful to learn as much as possible from the characteristic approach, which is less general, but works well. Third, we have started a number of smaller projects that allow us test what works and what does not in simplified situations, and actually start a mathematical analysis of the properties of our algorithms. We present some preliminary results below.

\section{GENERAL CONFORMAL FIELD EQUATIONS}

The approach suggested above and sketched in Fig. (1) is well adapted to computing gravitational wave signals, but can not reproduce a global representation of the spacetime, which includes spacelike infinity $\imath^{0}$. From the point of view of an observer at $\mathscr{I}^{+}$, $\imath^{0}$ represents the infinite past, which is clearly relevant for certain questions, e.g. a quasistationary solution may have persisted for a very long time, before violent dynamics sets in. Friedrich's general conformal field equations [13], which rely on the conformal Gauss gauge, allow for a global treatment, in which different asymptotic regions can be handled with one system of equations. Using this method can provide initial data for a 
hyperboloidal code that is actually determined from a Cauchy surface. Also, numerical experiments with this system might give rise to a better understanding of the regularity issues around spatial infinity.

As a first step we have used the general conformal field equations to construct the Schwarzschild-Kruskal solution with initial data specified on a Cauchy surface. Using the conformal Gauss gauge, in which by spherical symmetry all equations become ordinary differential equations, it was possible for the first time to cover the entire Schwarzschild-Kruskal spacetime including spacelike, null and timelike infinity and the domain close to the singularity (Fig. 2). These results can also be seen as a feasibility study of the conformal Gauss gauge. Current work is directed to the numerical solution of the general conformal field equations for non-spherically symmetric initial data.

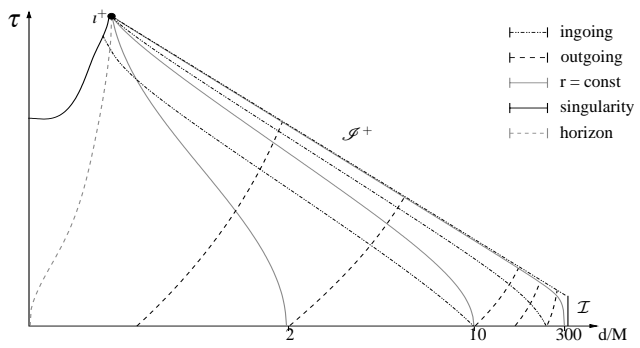

FIGURE 2. Schwarzschild-Kruskal spacetime in a conformal Gauss gauge.

\section{MINKOWSKI SPACE}

A natural first exercise when entering uncharted territory in NR is to consider Minkowski space. A comparison of evolutions of Minkowski space in various gauges with the full conformal field equations has been reported in [2], a particularly interesting case is, when $\mathscr{I}^{+}$is identified with a fixed coordinate sphere and the conformal geometry is chosen stationary. In this case a constraint violating continuum instability is found. Inspection of the equations suggests the instability to be due to the type of effect described in this section. Recently, the mathematical tools to clarify this have been discussed by Frauendiener and Vogel [16].

As a simple exercise for this type of problem, we have analyzed the case of a Maxwell field $\left(E^{a}, B^{a}\right)$ on Minkowski space sliced by non-trivial hypersurfaces:

$$
\begin{aligned}
& \partial_{t} E^{a}-\beta^{b} \partial_{b} E^{a}-\alpha \varepsilon^{a b c} h_{c d} \partial_{b} B^{d}=\alpha\left(\varepsilon^{a b c} h_{c d} \chi_{b} B^{d}-\chi E^{a}+\varepsilon^{a b c} h_{c d} \Gamma_{e b}^{d} B^{e}\right)-E^{b} \partial_{b} \beta^{a}, \\
& \partial_{t} B^{a}-\beta^{b} \partial_{b} B^{a}+\alpha \varepsilon^{a b c} h_{c d} \partial_{b} E^{d}=-\alpha\left(\varepsilon^{a b c} h_{c d} \chi_{b} E^{d}+\chi B^{a}+\varepsilon^{a b c} h_{c d} \Gamma_{e b}^{d} E^{e}\right)-B^{b} \partial_{b} \beta^{a}
\end{aligned}
$$

The constraints and constraint propagation equations then are

$$
\begin{array}{ll}
0=\mathcal{E}:=\partial_{b} E^{b}+\Gamma_{c b}^{b} E^{c}, & \partial_{t} \mathcal{E}-\beta^{a} \partial_{a} \mathcal{E}=-\alpha \chi \mathcal{E}, \\
0=\mathcal{B}:=\partial_{b} B^{b}+\Gamma_{c b}^{b} B^{c}, & \partial_{t} \mathcal{B}-\beta^{a} \partial_{a} \mathcal{B}=-\alpha \chi \mathcal{B} .
\end{array}
$$

Here, $h_{a b}, \chi, \chi_{a}, \Gamma_{b c}^{a}, \alpha$ and $\beta^{a}$ are 3-metric, mean extrinsic curvature, acceleration, Christoffel symbol of $h_{a b}$, lapse and shift respectively. From the constraint propagation 
equations one directly reads off a stability prognosis in the spirit of Frauendiener and Vogel [16]: if $\chi<0$, a constraint violating continuum instability has to be expected, whereas $\chi>0$ should result in constraint damping. Both effects will be demonstrated below for a simple class of slices in Minkowski space with a fixed sign of $\chi$. Note that densitizing the evolved fields can change the sign of the $\chi$ factors. This example thus demonstrates that one needs to be aware of a subtle interplay between the evolution system, choice of variables and gauge. Continuum instabilities of this type are essentially an ODE effect in the sense that they are determined by lower order source terms rather than spatial derivatives. Consequently, it is important to realize that for numerical purposes, analyzing the principal part is only a starting point. In general, lower order terms have to be carefully analyzed and a formulation of the theory has to be chosen that avoids instabilities. Clearly, this process benefits from avoiding excess baggage when formulating the equations one starts with.

In nonlinear situations, the decay of the fields is delayed by nonlinear interactions, and the ODE effects have an even stronger influence than in linear situations. In order to monitor these effects over a considerable amount of time, we consider a finite box with ideally conducting walls, i.e. a cavity in which the field excitation is reflected back and forth. We foliate Minkowski space with simple hyperboloids that are bent only in $\mathrm{X}$-direction and are flat in yz-directions: $\{t=$ const $\}$-surfaces with $t(T, X, Y, Z)=$ $T-\kappa\left(\sqrt{1+X^{2}}-1\right)$. For initial data we use analytically known eigenmodes of the cavity, transformed appropriately from standard Minkowski to the curved coordinates. The results of our experiments are presented in fig. 3 .


FIGURE 3. (a) Evolution in standard Minkowski coordinates; behaves nicely, energy conserved, linear drift away from exact solution (error energy norm depends linearly on time). Oscillations in the constraints due to lowered accuracy of constraint calculation at the boundary (stencil limitation). (b) Evolution in stable foliation $(\kappa=+1)$; behaves nicely, energy conserved, drift away from exact solution better than linear. (c) Evolution in unstable foliation $(\kappa=-1)$, behaves very badly, exponential growth of both constraints, exponential deviation from exact solution, finally triggers exponential growth of the energy.

\section{SOLUTION OF THE CONSTRAINTS}

Solving the constraints is interesting from two perspectives: first it is a necessary prerequisite for evolutions, and second, since a general procedure for solving the regular conformal constraints is not known, it provides an interesting example of a more ad-hoc regularization procedure for the Einstein equations. We consider an isotropic initial hypersurface, i.e. $\tilde{\chi}_{a b}=\tilde{\chi} \tilde{h}_{a b} / 3$ with $\tilde{\chi}=$ const. This ansatz solves the momentum constraint 
and is in some sense analogous to time symmetry for asymptotically euclidean slices. By applying the Lichnerowicz-York procedure to the rescaled metric $\Omega^{2} \tilde{h}_{\mu \nu}=\phi^{4} h_{\mu \nu}$, the Hamiltonian constraint is converted into the Yamabe equation

$$
4 \Omega^{2} D^{\mu} D_{\mu} \phi-4 \Omega D^{\mu} \Omega D_{\mu} \phi-\left(\frac{R}{2} \Omega^{2}+2 \Omega D^{\mu} D_{\mu} \Omega-3 D^{\mu} \Omega D_{\mu} \Omega\right) \phi=\frac{1}{3} \tilde{\chi}^{2} \phi^{5},
$$

where $D_{\mu}$ denotes the spatial covariant derivative and $R$ its Ricci scalar. For $\Omega \neq 0$ this is a semilinear elliptic equation, but its principal part vanishes on the conformal boundary and standard elliptic theory cannot be applied. The existence of smooth solutions $\phi$ has been proven in [17] under the condition that the extrinsic 2-curvature induced on the initial cut of $\mathscr{I}$ by the free metric is pure trace. The Yamabe equation then also determines the boundary values to be $\phi^{2}=3|\tilde{\chi}|^{-1} \sqrt{D^{\mu} \Omega D_{\mu} \Omega}$ on $\mathscr{I}$.

As an example, we consider the simple axisymmetric Brill ansatz

$$
d \sigma^{2}=e^{a q(\rho, z)}\left(d \rho^{2}+d z^{2}\right)+\rho^{2} d \varphi^{2}, \quad q(\rho, z)=\rho^{2} e^{-\left(\rho^{2}+z^{2}\right)} .
$$

Such data are well studied in the asymptotically euclidean regime where it is known that for small amplitudes $a$ the waves eventually disperse, leaving flat space behind, whereas for large values of $a$ the waves collapse and form trapped surfaces (in particular we have used such data to test our code against known results [18]). In the hyperboloidal case, the problem becomes nonlinear due to the non-vanishing of $\tilde{\chi}$, which we set to unity without restricting generality. Choosing the conformal gauge as $\Omega=1-r^{2}$ puts $\mathscr{I}$ to $r=1$ and makes the regularity condition on the extrinsic 2-curvature of $\mathscr{I}$ be identically satisfied. The resulting nonlinear boundary value problem can be simply discretized with $2^{\text {nd }}$ order finite differences and solved through a preconditioned GMRES method [19].

For the physical interpretation of the data it is interesting to search for marginally trapped surfaces, i.e. surfaces on which the null expansion $\Theta_{+}$vanishes. Note that since $\mathscr{I}^{+}$is a "surface at infinity", the expansions take the unique values $\Theta_{+}=\frac{4}{3} \tilde{\chi}, \Theta_{-}=0$ there. Since the geometry is euclidean in the vicinity of the axis, the expansions have their flat space behavior $\Theta_{ \pm} \rightarrow \pm \infty$ for $r \rightarrow 0$. Marginal surfaces can now develop if there exist values of the amplitude $a$ for which $\Theta_{+}$becomes non-positive in between. Surprisingly, while in the asymptotically euclidean case this happens generically, for the classes of data, we have studied, $\Theta_{+}$remains strictly positive and no trapped surfaces exist even for extremely high amplitudes.
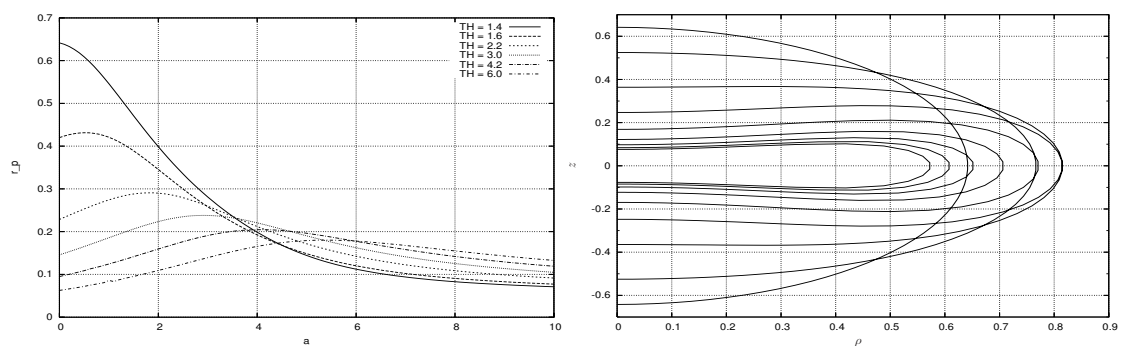

FIGURE 4. Left: Polar radii of equiexpansion surfaces for different amplitudes. Right: Shape of the surface $\Theta_{+}=1.4$ for $a=0$ (innermost), 3,6, .,24 (outermost). 


\section{CONCLUSIONS}

The prime motivation to study evolutions based on hyperboloidal slicings is that they enable us to reach null infinity with the flexibility of Cauchy codes. Using the example of the Maxwell equations we have also discussed that hyperboloidal hypersurfaces may tend to create either strong constraint damping or growth, which makes them interesting both as a model for what can go wrong and as a potential remedy. The general conformal field equations allow us to treat null and spacelike infinity in a unified picture, which we hope to help understand the physical significance of the idealizations one makes when using the compactified picture. In order to develop hyperboloidal codes that can handle physically interesting situations involving dynamical black holes and gravitational radiation, we believe it will be fruitful to obtain a fresh perspective on the compactification problem and consider adapted gauges as a starting point for regularizing equations rather than proceeding in the opposite direction.

\section{ACKNOWLEDGMENTS}

The authors thank C. Lechner for her work on computer algebra tools, especially those for the Maxwell equations, and I. Hinder for his continuing development work on Kranc. This work was supported in part by the SFB/Transregio 7 "Gravitational Wave Astronomy" of the German Science Foundation.

\section{REFERENCES}

1. J. Frauendiener, Living Rev. Relativity 7 (2004).

2. S. Husa, Lect. Notes Phys. 617, 159-192 (2003), gr-qc/0204057.

3. R. Penrose, Phys. Rev. Lett. 10, 66-68 (1963).

4. J. Frauendiener, Class.Quant.Grav. 17, 373-387 (2000), gr- qc / 9808072.

5. H. Friedrich, Proc. Roy. Soc. Lond. A375, 169-184 (1981).

6. N. T. Bishop, R. Gomez, L. Lehner, M. Maharaj, and J. Winicour, Phys. Rev. D56, 6298-6309 (1997), gr-qc/9708065.

7. Y. Zlochower, R. Gomez, S. Husa, L. Lehner, and J. Winicour, Phys. Rev. D68, 084014 (2003).

8. S. Husa, Phys. Rev. D54, 7311-7321 (1996), gr-qc/9606042.

9. S. Brandt, and B. Brügmann, Phys. Rev. Lett. 78, 3606-3609 (1997), gr- qc / 9703066.

10. S. Husa, Asymptotically flat initial data for gravitational wave spacetimes, conformal compactification and conformal symmetry, Ph.D. thesis, University of Vienna (1998).

11. B. Brügmann, Int. J. Mod. Phys. D8, 85-100 (1999), gr-qc/ 9708035.

12. F. Pretorius, Phys. Rev. Lett. 95, 211101 (2005), gr-qc/ 0507014.

13. H. Friedrich, 50 years of the Cauchy problem in general relativity (2004), gr-qc/0304003.

14. L. Andersson, Lect. Notes Phys. 604, 183-194 (2002), gr- qC/ 0205083.

15. S. Husa, I. Hinder, and C. Lechner (2004), gr- qc/ 0404023.

16. J. Frauendiener, and T. Vogel, Class. Quant. Grav. 22, 1769-1793 (2005), gr-qc/ 0410100.

17. L. Andersson, P. T. Chruściel, and H. Friedrich, Comm. Math. Phys. 149, 587-612 (1992).

18. M. Alcubierre, S. Brandt, B. Brügmann, C. Gundlach, J. Massó, E. Seidel, and P. Walker, Class. Quant. Grav. 17, 2159-2190 (2000), gr-qC/9809004.

19. Y. Saad, Iterative Methods for Sparse Linear Systems, SIAM, 2003, second edn. 